\title{
Identification of Blastocystis subtypes in clinical stool samples from Sao Paulo City, Brazil
}

\author{
GESSICA B. MELO ${ }^{1,2}$, FABIANA M. PAULA ${ }^{1,2}$, FERNANDA M. MALTA $^{2,3}$, \\ CELINA W. MARUTA ${ }^{4}$, PAUlO R. CRIADO ${ }^{4}$, VERA L. P. CASTILHO ${ }^{5}$, \\ ELENICE M. N. GONÇALVES ${ }^{5}$, MARIA C. ESPIRITO SANTO ${ }^{1}$ and \\ RONALDO C. B. GRYSCHEK ${ }^{1,2} *$ \\ ${ }^{1}$ Laboratório de Investigação Médica (LIM/06), Hospital das Clínicas da Faculdade de Medicina, \\ Universidade de São Paulo, São Paulo, Brazil \\ ${ }^{2}$ Instituto de Medicina Tropical, Universidade de São Paulo, São Paulo, Brazil \\ ${ }^{3}$ Laboratório de Investigação Médica (LIM/07), Hospital das Clinicas da Faculdade de Medicina, \\ Universidade de São Paulo, São Paulo, Brazil \\ ${ }^{4}$ Departamento de Dermatologia, Hospital das Clínicas da Faculdade de Medicina, Universidade de São Paulo, \\ São Paulo, Brazil \\ ${ }^{5}$ Seção de Parasitologia, Divisão do Laboratório Central do Hospital das Clínicas da Faculdade de Medicina, \\ Universidade de São Paulo, São Paulo, Brazil
}

(Received 31 August 2016; revised 7 February 2017; accepted 12 February 2017)

\begin{abstract}
SUMMARY
Blastocystis sp. is a protozoan commonly found in human and animal stool samples. Several pathogenic and zoonotic aspects of this organism are still unknown. The aim of the present study was to investigate Blastocystis subtypes (STs) in samples from patients of the Hospital das Clínicas of the Faculdade de Medicina at the Universidade de São Paulo (HC-FMUSP), Brazil. Blastocystis sp.-positive stool samples diagnosed at the Section of Parasitology of the Central Laboratory (HC-FMUSP) were used for DNA isolation. Polymerase chain reaction (PCR) was performed using specific primers targeting the small-subunit rRNA gene. Direct DNA sequencing of the PCR products was performed and the DNA sequences were then aligned and compared with other sequences obtained from the GenBank database. Phylogenetic analysis was used to identify STs and determine the phylogenetic relationships between the sequences. Four STs were identified: ST1 (22.5\%), ST2 (12.5\%), ST3 (60\%) and ST6 (5\%). In conclusion, ST3 was the most prevalent ST among the human isolates followed by ST1. The present study is one of the few providing STs data from the human population in South America. Determining ST prevalence in human samples may contribute to the monitoring of Blastocystis sp. infection transmission in endemic regions.
\end{abstract}

Key words: Blastocystis, subtypes, Brazil.

\section{INTRODUCTION}

The high number of samples positive for Blastocystis $\mathrm{sp}$, , an organism that was rarely reported in routine parasitological diagnosis until three decades ago, has received increasing attention in recent years. Although, the pathogenic role of Blastocystis sp. is controversial, accumulating evidence from recent studies supports the idea that they constitute an emerging pathogen (Tan, 2008; Tan et al. 2010). This enigmatic organism has also been identified in a wide range of animals. The taxonomic status of Blastocystis sp. has remained elusive until recently; phylogenetic studies based on small-subunit ribosomal DNA (SSU rRNA) have demonstrated that it is a member of phylum Stramenopiles (Silberman et al. 1996; Santín et al. 2011).

* Corresponding author: Laboratório de Investigação Médica (LIM-06), Hospital das Clínicas da Faculdade de Medicina, USP, São Paulo, Brazil and Instituto de Medicina Tropical, prédio II, $2^{\circ}$ andar, Av. Dr Enéas de Carvalho Aguiar, 470, Cerqueira Cesar, 05403-000, São Paulo, SP, Brazil. E-mail: rcbgry@usp.br
Blastocystis sp. is usually detected using conventional parasitological methods such as the direct method, concentration method with formalin-ether and stained smears (Stensvold et al. 2008; Tan, 2008). Culturing methods have been regarded as the gold standard for the detection of Blastocystis sp., because although these methods show high sensitivity (Yoshikawa et al. 2011; Zhang et al. 2012). Polymerase chain reaction (PCR) amplification of Blastocystis DNA from cultures or feces is thought to be the most sensitive detection method (Tan, 2008). DNA-based methods have been developed to identify genetic variation between Blastocystis sp. forms that are morphologically indistinguishable using a microscope (Alfellani et al. 2013). Molecular studies have focused predominantly on determining the prevalence of Blastocystis subtypes (STs), as well as possible factors associated with pathogenicity (Tan et al. 2010). Studies using the sequence analysis of Blastocystis sp. small subunit ribosomal RNA genes (SSU rDNA) have demonstrated that there are approximately 17 different lineages, termed subtypes (ST1-ST17), isolates from mammals and birds 
(Wawrzyniak et al. 2013). Human isolates have been shown to have a higher occurrence of STs 1-4, with limited presence of STs 5-9 (Stensvold et al. 2007; Scanlan, 2012; Alfellani et al. 2013).

The molecular epidemiology of Blastocystis sp. infections remains unknown in many parts of the world. Recent studies have provided further information regarding ST distribution among human populations (Tan, 2008; Alfellani et al. 2013); however, very few studies have been conducted in Latin America (Santín et al. 2011). To date, a small number of studies subtyping Blastocystis (Malheiros et al. 2011; David et al. 2015; Ramírez et al. 2016) have been conducted in Brazil. The aim of this study was to investigate the prevalence of Blastocystis STs in clinical stool samples from the Hospital das Clínicas of the Faculdade de Medicina de São Paulo (HC-FMUSP), Brazil.

\section{MATERIALS AND METHODS}

\section{Study population}

This study was approved by the local Research Ethics Committee of the Hospital das Clínicas of the Faculdade de Medicina de São Paulo under protocol n ${ }^{\circ} .488 \cdot 701$. Sixty Blastocystis sp.-positive stool samples were selected based on the positivity in routine parasitological examinations conducted at the Section of Parasitology, Central Laboratory, HCFMUSP, Brazil. The techniques employed at this laboratory include the Faust method, Lutz method and permanent-stained smears (Garcia, 2001), considering positivity in at least one of the parasitological methods. Fifteen Blastocystis sp.-negative stool samples, determined by the parasitological methods, and 15 stool samples positive for other parasite infections (Ascaris lumbricoides, Entamoeba histolytica, Giardia lamblia, Endolimax nana and Entamoeba coli) were used as controls. Once the parasitological techniques were performed, the stool samples were aliquoted and stored at $-20^{\circ} \mathrm{C}$ for subsequent DNA isolation.

\section{DNA extraction}

Approximately $200 \mathrm{mg}$ of stool sample were washed twice with phosphate-buffered saline $\left(0.01 \mathrm{M} \mathrm{L}^{-1}\right.$, $\mathrm{pH} 7 \cdot 2)$. DNA was extracted from the pellet using the commercial QIAamp ${ }^{\circledR}$ DNA stool MiniKit (QIAGEN, Hilden, Germany), according to the manufacturer's instructions. DNA was eluted in $100 \mu \mathrm{L}$ of elution buffer and quantified using a NanoDrop ND1000 UV-VIS spectrophotometer v.3.2.1 (NanoDrop Technologies, Wilmington, DE, USA).

\section{DNA amplification and sequencing}

DNA amplification was performed by PCR using the primers RD5 (5'-ATC TGG TTG ATC CTG
CCAG T-3') and BhRDr (5'-GAG CTT TTT AAC TGC AAC AAC G-3'), located on SSU rRNA, as described by Scicluna et al. (2006); these primers amplify a $\sim 600$ bp fragment. PCR were performed in a $10 \mu \mathrm{L}$ volume containing $\sim 50 \mathrm{ng} \mu \mathrm{L}^{-1}$ of DNA, $2.0 \mu \mathrm{g}$ BSA, $0.2 \mathrm{~mm}$ each dNTP, $1.5 \mathrm{~mm}$ $\mathrm{MgCl}_{2}, 2 \mathrm{pM}$ of each primer, $1 \times$ PCR buffer and $1.25 \mathrm{U}$ GoTaq $^{\circledR}$ DNA Polymerase (Promega Corporation, Madison, USA). PCR amplification was conducted with a Master cycler ep gradient $\mathrm{S}$ thermocycler (Eppendorf, Hamburg, Germany) with the following conditions: initial denaturation step at $94{ }^{\circ} \mathrm{C}$ for $2 \mathrm{~min} ; 30$ cycles of $94{ }^{\circ} \mathrm{C}$ (denaturation) for $1 \mathrm{~min}, 61^{\circ} \mathrm{C}$ (annealing) for $1 \mathrm{~min}$ and $72{ }^{\circ} \mathrm{C}$ for $1 \mathrm{~min}$ (extension); and a final extension step of $72{ }^{\circ} \mathrm{C}$ for $2 \mathrm{~min}$. The PCR products were loaded on a $2 \%$ agarose gel containing Syber safe (Invitrogen ${ }^{\mathrm{TM}}$, Thermo Fisher Scientific Corporation, Waltham, USA) and subjected to electrophoresis in $1 \times$ TAE (Tris-acetate-EDTA) buffer. Positive and negative controls (DNA from positive cultures and PCR mix without DNA template, respectively) were included in each round of amplification.

PCR products were purified using of the ExoSAP enzyme (GE Healthcare, Piscataway, NJ, USA), according to the manufacturer's instructions, and then submitted to a sequencing reaction for incorporation of labelled ddNTPs (dideoxynucleotides) using the ABI PRISM ${ }^{\circledR}$ BigDyeTM Terminator kit (Applied Biosystems, Thermo Fisher Scientific Corporation, Waltham, USA). Direct DNA sequencing was performed using an automated ABI 3500 sequencer (Applied Biosystems).

\section{Positive controls}

The positive controls consisted of approximately $200 \mathrm{mg}$ of Blastocystis sp.-positive stool samples cultured as described by Zerpa et al. (2000). The cultures were maintained at $37^{\circ} \mathrm{C}$ and the pellets obtained by centrifugation at $12,400 \boldsymbol{g}$ for 1 min were examined using Lugol staining smear with optical microscopy (Olympus CX 41) after 24 and $48 \mathrm{~h}$.

For control testing of extracted DNA, all samples without amplification were tested using universal primers (forward: 18SEUDIR 5'-TCTGCCCTAA CTACTTTCGATGG-3' and reverse: 18SEUINV 5'-TAATTTGGCCTGCGCCTG-3') that amplify a $140-$ bp region of the eukaryotic $18 \mathrm{~S}$ ribosomal RNA gene, as described by Wang et al. (2013).

\section{Subtyping analysis}

Sequence analysis was performed using BioEdit software (Biological Sequence Alignment Editor http://www.mbio.ncsu.edu/bioedit/page2.html). Sequence alignments were conducted using CLUSTAL W and aligned with Blastocystis 
Table 1. Comparison of Parasitological methods and conventional PCR (cPCR) for detection of Blastocytis in the stool samples

\begin{tabular}{|c|c|c|c|c|c|c|c|c|c|}
\hline \multirow[b]{3}{*}{$\mathrm{cPCR}$} & \multicolumn{9}{|c|}{ Parasitological methods } \\
\hline & \multicolumn{3}{|c|}{ Faust } & \multicolumn{3}{|c|}{ Lutz } & \multicolumn{3}{|c|}{ Permanent-stained smears } \\
\hline & $(+)$ & $(-)$ & Total & $(+)$ & $(-)$ & Total & $(+)$ & $(-)$ & Total \\
\hline$(+)$ & 5 & 42 & 47 & 17 & 30 & 47 & 43 & 4 & 47 \\
\hline$(-)$ & 0 & 13 & 13 & 4 & 9 & 13 & 9 & 4 & 13 \\
\hline Total & 5 & 55 & 60 & 21 & 39 & 60 & 52 & 8 & 60 \\
\hline
\end{tabular}

sequences from GenBank (http://www.ncbi.nlm. nih.gov/GenBank/tbl2asn2). Phylogenetic analyses were performed using MEGA 5 and the neighbourjoining method with 1000 bootstrap replication for phylogenetic tree construction. The Proteromonas lacertae sequence (accession number U37108) was used as the outgroup. Sequences were compared with Blastocystis SSU rDNA sequences available from the National Center for Biotechnology Information (NCBI) using the BLAST program (Basic Local Alignment Search Tool). Additionally, Blastocystis STs were identified by determining the exact match or closest similarity against all known Blastocystis STs using www.pubmlst.org/blastocystis.

\section{RESULTS}

Of the 60 clinical stool samples positive for Blastocystis sp. by parasitological methods, 47 were positive by PCR (78\% positivity). PCR positivity was higher compared with other parasitological methods: $8 \%$ positivity by Faust, $35 \%$ by Lutz and lower than permanent-stained smears (87\%) (Table 1). The expected DNA fragment ( 600 bp) was detected in all PCR-positive samples and no DNA amplification of the target fragment was observed in the negative samples and stool samples positive for other parasite infections.

All PCR positive samples were sequenced and those that obtained good quality $(n=40)$ were compared with Blastocystis sequences deposited in the GenBank database (BLAST) and exhibited 98-100\% similarity. Seven sequences were excluded from the analysis, since they presented low-quality possibly due to poor amplification of DNA.

The phylogenetic analysis was performed and four different STs were identified (Fig. 1). ST1 was found in nine samples $(22 \cdot 5 \%)$, ST2 was present in five samples $(12 \cdot 5 \%)$, ST3, the most common ST, was found in 24 samples $(60 \%)$ and ST6 was present in two samples (5\%). Based on information available at www.pubmlst.org/blastocystis, three distinct alleles were identified within ST3 (alleles 34, 36 and 37), two in ST2 (alleles 12 and 71) and one in ST1 and ST6, corresponding to alleles 4 and 134 , respectively (Table 2 ).
DISCUSSION

Blastocystis sp. is the most common intestinal parasites found in human feces and are considered an emerging parasite with a worldwide distribution (Tan, 2008). The clinical features associated with blastocystosis range from non-specific intestinal symptoms to cutaneous disorders and the severity of these diseases varies from acute to chronic infections, a possible reason for these differences is genetic diversity (Tan, 2008; Tan et al. 2010; Scanlan, 2012). Although to date, no specific association has been observed between clinical findings and Blastocystis S'Ts (Özyurt et al. 2008).

A variety of parasitological techniques can be used to detect Blastocystis sp. in clinical samples (Tan, 2008). However, this technique has low sensitivity, leading to misdiagnosis or underestimation of the true prevalence of Blastocystis sp. (Scanlan, 2012; Alfellani et al. 2013). Moreover, this parasite appears in polymorphic forms and variable size in stool samples, further hindering its diagnosis. PCR is an alternative method used to detect Blastocystis sp. in epidemiological studies, offering increased sensitivity and specificity as well as rapid diagnosis (Parkar et al. 2007). The results of the present study demonstrate that PCR was more efficient than the Faust and Lutz methods in the detection of Blastocystis sp. Another advantage of using molecular techniques compared with conventional methods is that they can provide more information in terms of genetic variability and zoonotic relationship (Parkar et al. 2007).

In this study, 60 samples were diagnosed as Blastocystis sp. positive using parasitological technical (considered the Faust method, Lutz method and permanent-stained smears), while the PCR technique confirmed the presence of the DNA parasite in only 47 stool samples. Possible reasons for this difference are PCR inhibition stool samples, degradation of DNA due to extended storage time, or misidentification by light microscopy (Forsell et al. 2012; Mattiucci et al. 2016). However, negative PCR results were not caused by inhibition of the amplification by fecal components, because amplification using universal primers was observed in all samples. 


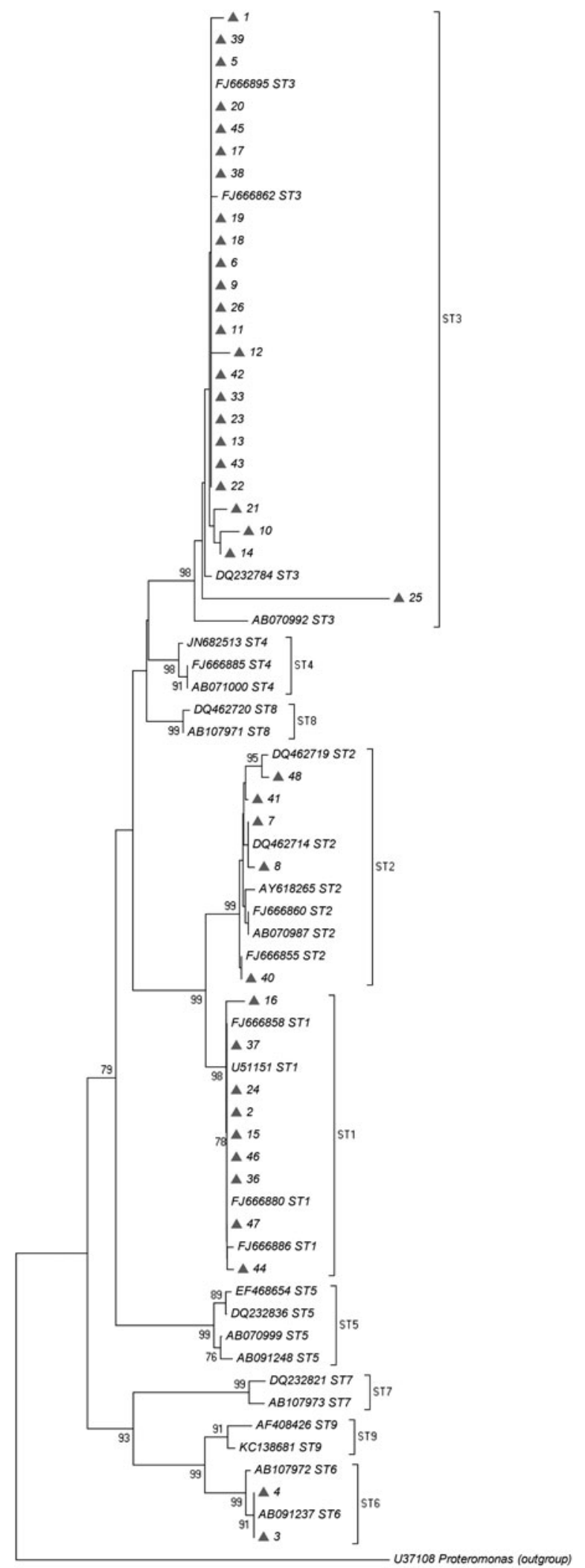

$\stackrel{\square .02}{1}$

Fig. 1. Phylogenetic analysis of Blastocystis sp. SSU rDNA sequences $(\sim 600 \mathrm{bp})$ generated in this study (identified by triangles) and reference sequences from GenBank (identified by accession number and subtypes). The phylogenetic tree was constructed using the neighbour-joining method. Bootstrap values are based on 1000 replicates. Bootstrap values of $<70 \%$ are not shown.
The suggested criteria for reporting infection intensity by parasitological methods are the observation of five or more parasites per high-powered field $(\times 400)$ for wet mounts or under oil immersion $(\times 1000)$ using permanent-stained smears (Tan, 2008). Other studies have included more detailed reports of parasite abundance (Leder et al. 2005; Özyurt et al. 2008) and quantified parasite abundance using definitions such as rare (one to two parasites in every 10 high-power fields), few to moderate (one parasite in every one to five high-power fields), and abundant (five or more parasites per high-power field) (Leder et al. 2005). In the present study, 13 of the 60 Blastocystis sp. positive samples identified using parasitological methods failed to produce Blastocystis-specific bands by PCR, five of which were reported as rare, four as few to moderate and four as suggestive forms by parasitological analyses. Mattiucci et al. (2016) have suggested that negative PCR results may be due to the amount of Blastocystis DNA being lower than the detection level.

Blastocystis in mammals and birds are subdivided into 17 STs, nine of which (ST1-ST9) have been found in humans (Stensvold and Clark, 2016). In 2007, a consensus of Blastocystis terminology was proposed, based in comparison with representative sequences of all known STs (Stensvold et al. 2007). The ST distribution in the present study was quite similar to that found in other countries. Indeed, the studies reported thus far indicate that the majority of human infections with Blastocystis sp. are attributable to ST3 isolates (Özyurt et al. 2008; Meloni et al. 2011; Forsell et al. 2012; Ramírez et al. 2016), as was the case in the present study $(60 \%)$. It is suggested that dominance of ST3 may be related to its human origin (Özyurt et al. 2008; Meloni et al. 2011; Yoshikawa et al. 2016).

An analysis of samples from indigenous regions of Brazil conducted by Malheiros et al. (2011) showed different results from those of the current study; ST1 was the dominant ST identified (41\%). It is possible that ST distribution is affected by the ethnic origin of the population and the limited contact between indigenous groups and people in other communities. ST1 and ST2 are also common in different regions in human isolates, while the other STs are found only sporadically (Alfellani et al. 2013). It is important to note the absence of ST4 in our study, similar to the results of other studies conducted in South America (Malheiros et al. 2011; David et al. 2015). In contrast, ST4 is observed with high frequency in continental Europe and the UK (Forsell et al. 2012; Alfellani et al. 2013; Mattiucci et al. 2016).

Another important finding of this study is the presence of ST6, rarely detected in human isolates. ST6 is considered as an avian ST, which points to a possible zoonotic origin (Mattiucci et al. 2016). 
Table 2. Distribution of Blastocystis SSU rDNA alleles retrieved from the samples on each subtytpes

\begin{tabular}{lccl}
\hline \hline Subtypes & $n(\%)$ & Alleles & Sample $^{\mathrm{a}}$ \\
\hline ST1 & $9 / 40(22 \cdot 5)$ & $4(9 / 9)$ & $2,15,16,24,36,37,44,46,47$ \\
ST2 & $5 / 40(12 \cdot 5)$ & $12(4 / 5)$ & $7,8,40,41$ \\
& & $71(1 / 5)$ & 48 \\
ST3 & $24 / 40(60 \cdot 0)$ & $34(11 / 24)$ & $5,6,10,11,12,17,23,26,33,42,45$ \\
& & $36(11 / 24)$ & $1,9,13,14,18,19,20,21,22,25,38$ \\
ST6 & $37(2 / 24)$ & 3,43 \\
\hline \hline
\end{tabular}

a Corresponds to the number of samples identified on the phylogenetic tree.

The reported differences in the number of STs identified in human populations as well as their relative abundance might indicate different reservoirs and transmission routes (Noël et al. 2005; Meloni et al. 2011).

In the present study, alleles 34,36 and 37 were detected in ST3. This is in agreement with observations reported by David et al. (2015) and Mattiucci et al. (2016). In contrast to our findings, Ramírez et al. (2016) showed a high diversity of alleles from ST1, ST2 and ST3. It is important to highlight that the comparison SSU rDNA alleles within the same ST can help determining the differences between the strains, which may possibly contribute to the identification of the potential for zoonotic transmission and pathogenic strains.

The present study is one of the few to analyse the incidence of Blastocystis STs in South America. Thus, our results are very important for understanding the geographic distribution of STs in Latin America. Moreover, these findings provide an initial molecular analysis that will enable future examination of unknown epidemiological aspects.

\section{ACKNOWLEDGEMENTS}

We would like to thank all the patients who provided the stool samples used in this study. We are very grateful to Ms Magali Orban for her technical assistance in conducting the parasitological analysis of fecal samples.

\section{FINANCIAL SUPPORT}

This research was supported by Fundação de Amparo à Pesquisa do Estado de São Paulo (FAPESP 2015/18213-6), Brazil.

\section{REFERENCES}

Alfellani, M., Stensvold, C. R., Vidal-Lapiedra, A., Uche Onuoha, E., Fagbenro-Beyioku, A. and Clark, G. (2013). Variable geographic distribution of Blastocystis subtypes and its potential implications. Acta Tropica 126, 11-18.

David, E. B., Guimarães, S., Oliveira, A. P., Oliveira-Siqueira, T. C. G., Bittencourt, G. N., Nardi, A. R. M., Ribolla, P. E. M., Franco, R. M. B., Branco, N., Tosini, F., Bella, A., Pozio, E. and Cacciò, S. M. (2015). Molecular characterization of intestinal protozoa in two poor communities in the State of São Paulo, Brazil. Parasite \& Vectors 8, 103-115.
Forsell, J., Granlund, M., Stensvold, C. R., Clark, G. C. and Evengard, B. (2012). Subtype analysis of Blastocystis isolates in Swedish patients. European Fournal of Clinical Microbiology Infectious Diseases 31, 1689-1696.

Garcia, L. S. (2001). Diagnostic Medical Parasitology, 4th Edn. Washington, DC: American Society for Microbiology.

Leder, K., Hellard, M., Sinclair, M. I., Fairley, C. K. and Wolfe, R.

(2005). No correlation between clinical symptoms and Blastocystis hominis in immunocompetent individuals. Fournal of Gastroenterology and Hepatology 20, 1390-1394.

Malheiros, A. F., Stensvold, C. R., Clark, C. G., Braga, G. B. and Shaw, J. J. (2011). Short report: molecular characterization of Blastocystis obtained from members of the indigenous Tapirapé ethnic group from the Brazilian Amazon region, Brazil. The American fournal of Tropical Medicine and Hygiene 85, 1050-1053.

Mattiucci, S., Crisafi, B., Gabrielli, S., Paoletti, M. and Cancrini, G. (2016). Molecular epidemiology and genetic diversity of Blastocystis infection in humans in Italy. Epidemiology and Infection 144, 635-646.

Meloni, D., Sanciu, G., Poirier, P., El Alaoui, H., Chabé, M., Delhaes, L., Dei-Cas, E., Delbac, F., Luigi Fiori, P., Di Cave, D. and Viscogliosi, E. (2011). Molecular subtyping of Blastocystis sp. isolates from symptomatic patients in Italy. Parasitology Research 109, 613-619.

Noë1, C., Dufernez, F., Gerald, D., Edgcomb, V. P., DelgadoViscogliosi, P., Ho, L. C., Singh, M., Wintjens, R., Sogin, M. L., Capron, M., Pierce, R., Zenner, L. and Viscogliosi, E. (2005). Molecular phylogenies of Blastocystis isolates from different hosts: implications for genetic diversity, identification of species, and zoonosis. Fournal of Clinical Microbiology 43, 348-355.

Özyurt, M., Kurt, O., Mølbak, K., Nielsen, H. V., Haznedaroglu, T. and Stensvold, C. R. (2008). Molecular epidemiology of Blastocystis infections in Turkey. Parasitology International 57, 300-306.

Parkar, U., Traub, R. J., Kumar, S., Mungthin, S., Vitali, S., Leelayoova, S., Morris, K. and Thompson, R. C. (2007). Direct characterization of Blastocystis from faeces by PCR and evidence of zoonotic potential. Parasitology 134, 359-367.

Ramírez, J. D., Sánchez, A., Hernández, C., Flórez, C., Bernal, M. C., Giraldo, J. C., Reyes, P., López, M. C., García, L., Cooper, P. J., Vicuña, Y., Mongi, F. and Casero, R. D. (2016). Geographic distribution of human Blastocystis subtypes in South America. Infection Genetics and Evolution 41, 32-35.

Santín, M., Gómez-Muñoz, M. T., Solano Aguilar, G. and Fayer, R. (2011). Development of a new PCR protocol to detect and subtype Blastocystis spp. From humans and animals. Parasitology Research 109, 205-212.

Scanlan, P. D. (2012). Blastocystis: past pitfalls and future perspectives. Trends in Parasitology 28, 327-334.

Scicluna, S. M., Tawari, B. and Clark, C. G. (2006). DNA barcoding of Blastocystis. Protist 157, 77-85.

Silberman, J. D., Sogin, M. L., Leipe, D. D. and Clark, C. G. (1996). Human parasite finds taxonomic home. Nature 380, 398.

Stensvold, C. R. and Clark, C. G. (2016). Current status of Blastocystis: a personal view. Parasitology International 65, 763-771.

Stensvold, C. R., Suresh, G. K., Tan, K. S., Thompson, R. C., Traub, R. J., Viscogliosi, E., Yoshikawa, H. and Clark, C. G. (2007). Terminology for Blastocystis subtypes - a consensus. Trends in Parasitology 23, 93-96.

Stensvold, C. R., Nielsen, H. V., Mølbak, K. and Smith, H. V. (2008). Pursuing the clinical significance of Blastocystis - diagnostic limitations. Trends in Parasitology 25, 23-29. 
Tan, K. S. (2008). New insights on classification, identification and clinical relevance of Blastocystis spp. Clinical Microbiology Reviews 21, 639-665. Tan, K. S., Mirza, H., Teo, J. D., Wu, B. and Macary, P. A. (2010). Current views on the clinical relevance of Blastocystis spp. Current Infectious Disease Reports 12, 28-35.

Wang, W., Cuttell, L., Bielefeldt-Ohmann, H., Inpankaew, T., Owen, H. and Traub, R. J. (2013). Diversity of Blastocystis subtypes in dogs in different geographical settings. Parasite $\mathcal{F}^{\circ}$ Vectors 6, 215-219.

Wawrzyniak, I., Poirier, P., Viscogliosi, E., Dionigia, M., Texier, C., Delbac, F. and El Alaoui, H. (2013). Blastocystis, an unrecognized parasite: an overview of pathogenesis and diagnosis. Therapeutic Advances in Infectious Disease 1, 167-178.
Yoshikawa, H., Dogruman-AI, F., Turk, S., Kustimur, S., Balaban, N. and Sultan, N. (2011). Evaluation of DNA extraction kits for molecular diagnosis of human Blastocystis subtypes from fecal samples. Parasitology Research 109, 1045-1050.

Yoshikawa, H., Koyama, Y., Tsuchiya, E. and Takami, K. (2016). Blastocystis phylogeny among various isolates from humans to insects. Parasitology International 65, 750-759.

Zerpa, R. L., Huichol, L., Náquira, C. and Espinoza, I. (2000). A simplified culture method for Blastocystis hominis. Revista Mexicana de Patología Clínica 47, 17-19.

Zhang, X., Qiao, J., Wu, X., Da, R., Zhao, L. and Wei, Z. (2012). In vitro culture of Blastocystis hominis in three liquid media and its usefulness in the diagnosis of blastocystosis. International Yournal of Infectious Diseases 16, 23-28. 